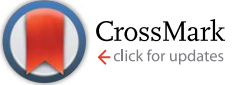

Cite this: RSC Adv., 2015, 5, 48290

Received 26th February 2015

Accepted 26th May 2015

DOI: $10.1039 / \mathrm{c} 5 \mathrm{ra03499j}$

www.rsc.org/advances

\section{Cellulose acetate graft-(glycidylmethacrylate-g- PEG) for modification of AMC ultrafiltration membranes to mitigate organic fouling}

\begin{abstract}
Ayyavoo Jayalakshmi, ${ }^{a}$ In-Chul Kim*b and Young-Nam Kwon*a
A hydrophilic cellulose acetate-graft-(glycidylmethacrylate-g-polyethylene glycol) (CA-g-(GMA-g-PEG)) was synthesized and incorporated into acetylated methyl cellulose (AMC) to prepare antifouling ultrafiltration membranes. The successful synthesis of CA-g-(GMA-g-PEG) was confirmed by ${ }^{1} \mathrm{H}-\mathrm{NMR}$ and $\mathrm{X}$-ray photoelectron spectroscopy studies. The AMC blend membranes were characterized by $\mathrm{X}$-ray diffraction (XRD), thermo gravimetric analysis (TGA), scanning electron microscopy (SEM), atomic force microscopy (AFM), confocal laser scanning microscopy (CLSM) and contact angle analysis to investigate the effect of CA-g-(GMA-g-PEG) on the properties of the membrane surface. The increase of CA-g(GMA-g-PEG) content in the AMC matrix reduced the macrovoids and transformed to a sponge-like structure in the entire membrane cross section. Furthermore, the increase in the graft moiety enhanced the performance of the membranes. Surface free energy parameters calculated from the contact angle measurements indicate that the interfacial free energy of the blend membranes were lower than those of the pure AMC membranes. The modified membrane surface became more hydrophilic and more wettable because of the preferential orientation of these polar groups towards the membrane surface. The efficiency of these membranes in the separation of singlet foulants and multi foulants increased significantly, thus increasing the fouling resistance. These membranes would be useful for organic fouling prevention during water and wastewater treatment.
\end{abstract}

\section{Introduction}

Membrane technology has been progressively used for the elimination of organic foulants from surface and ground waters because of its smaller footprint and reliable effluent quality. ${ }^{1-3}$ In particular, ultrafiltration (UF) membranes are promising cost-effective alternatives to conventional energy intensive separation processes. As a result, they are widely used in the pretreatment process for nanofiltration (NF) and reverse osmosis (RO) and as a key component for membrane bioreactors. ${ }^{4,5}$ In spite of the potential effectiveness, organic fouling and the subsequent flux decline of the UF membranes during the operation still remain issues to be solved. Organic fouling of the membranes in the water treatment is inevitably increasing operation and maintenance costs. Typically, natural water contains multifoulants (such as polysaccharides, humic and protein-like substances) rather than single well-defined ones. Many researchers have demonstrated that the binary and

\footnotetext{
${ }^{a}$ School of Urban and Environmental Engineering, Ulsan National Institute of Science and Technology, Ulsan 689-798, Republic of Korea. E-mail: kwonyn@unist.ac.kr; Tel: $+82-52-217-2810$

${ }^{b}$ Environment \& Resources Research Center, Korea Research Institute Chemical Technology, Daejeon 305-606, Republic of Korea. E-mail: ickim@krict.re.kr; Tel: $+82-42-860-7638$
}

ternary foulant systems are more complex than individual foulants. ${ }^{6-8}$

The interaction between the membrane surface and the foulant is a critical factor in membrane fouling. Therefore, several approaches have been taken to diminish the membrane fouling by varying the surface charge or increasing the surface hydrophilicity discourage the attachment and/or adsorption of foulants onto the surface. ${ }^{9-11}$ Blending of polymers with superior characteristics at the membrane manufacturing step can improve the surface properties and subsequent filtration efficiency and fouling resistance.

Acetylated methyl cellulose (AMC) has been currently reported as a new membrane material with excellent film forming properties useful for UF and RO process. ${ }^{12,13}$ Cellulose acetate (CA) also has superior polymeric properties including high salt rejection, moderate flux and renewable source of raw material and can go into various applications because of its advantages. ${ }^{\mathbf{1 4 , 1 5}}$ However, the application of these cellulosebased materials is severely limited because of the dense skin layer and subsequent lower flux. The denser skin layer formation is caused by slow diffusion of non-solvent towards casting solution and the subsequent coagulation retardation during the immersion precipitation step. Thus, the polymer modification becomes necessary prior to the membrane fabrication. The superior fouling resistance and separation efficiency of CA 
membranes can be achieved by surface graft polymerization with monomers such as polyacrylonitrile (PAN), polyvinylpyrrolidone (PVP), pluronic F127 and polyethylene glycol methacrylate (PEGMA) ${ }^{16-18}$ Jiang et al. reported that the CA-gPAN ultrafiltration membrane prepared by the phase inversion method showed superior oil fouling resistance under high operating pressure. ${ }^{19}$

Herein, we report a new research effort to improve the selectivity and permeability of CA by grafting glycidyl methacrylate (GMA) and PEG 200 in series on the CA backbone using CAN initiator and $n$-butyl lithium and prepare CA- $g$-(GMA- $g$ PEG) incorporated AMC ultrafiltration membranes. An extensive literature survey indicated that this is the first attempt to investigate the usage of hydrophilic CA-g-(GMA-g-PEG) for the modification of hydrophilic AMC membranes. Furthermore, the effect of AMC/CA- $g$-(GMA- $g$-PEG) blend membranes on the rejection of multifoulants such as humic acid (HA), sodium alginate (SA) and bovine serum albumin (BSA) has been investigated. The performance of the pure AMC and AMC blend membranes was evaluated by filtration experiments in which AMC/CA- $g$-(GMA- $g$-PEG) blend membranes were compared to the unmodified AMC membranes in terms of permeability, surface morphology, surface roughness, hydrophilicity, fouling resistance, and flux recovery after washing. The successful developments would allow the safe and economical advancement in the preparation of antifouling AMC blend membranes for UF applications.

\section{Experimental}

\subsection{Chemicals and reagents}

Acetylated methyl cellulose (MW - $300 \mathrm{kDa}$ ) was supplied by KRICT, Korea. Cellulose acetate (MW - $30 \mathrm{kDa}$ ) was purchased from Sigma-Aldrich (MO, USA) and used without any pretreatment. $N, N$-Dimethylformamide (DMF), $N$-methyl-2-pyrrolidone (NMP), ammonium cerium(Iv) nitrate (Ce salt), glycidylmethacrylate (GMA), PEG 200 and methanol were purchased from Daejung Chemicals \& Metals Co., Ltd., Korea and were used as received. Analytical grade butyl lithium solution (1.6 $\mathrm{M}$ in hexane) was purchased from Sigma-Aldrich, Korea. Non-woven fabric (NWF) used as the membrane support was supplied by Toray Chemical Korea.

Sodium lauryl sulfate (SLS) was obtained from Daejung Chemicals \& Metals Co., Ltd., Korea, and used as a surfactant in the coagulation bath. SA, BSA and sodium phosphate buffer were obtained from Sigma Aldrich, Korea and used as received. HA was obtained from Alfa Aesar, Korea and used as a model compound for natural organic matter in drinking water. The $\mathrm{pH}$ of the solution was adjusted to the desired value using either $\mathrm{HCl}$ or $\mathrm{NaOH}$ as needed.

\subsection{Synthesis of CA- $g$-(GMA- $g$-PEG)}

The direct grafting of GMA onto CA was performed via free radical polymerization using cerium ion initiator in the polymer solution. $8 \mathrm{~g}$ of CA powder was dissolved in $100 \mathrm{~mL}$ DMF with agitation under nitrogen atmosphere. After the elimination of oxygen by continuous bubbling of nitrogen gas, $1.5 \mathrm{~g}$ of Ce salt dissolved in 1.0 $\mathrm{M}$ nitric acid solution was added to the reaction vessel as initiator. The mixture was stirred at room temperature for $\sim 30 \mathrm{~min}$ and then, $4 \mathrm{~mL}$ of GMA monomers was added in the flask. The graft polymerization was performed at $30{ }^{\circ} \mathrm{C}$ for $12 \mathrm{~h}$ with gentle stirring.

The resulting polymer solution was cooled to $-40{ }^{\circ} \mathrm{C}$, and $2 \mathrm{~mL}$ of $n$-butyllithium solution, (1.6 M in hexane) was added drop wise with vigorous stirring. After stirring for another $30 \mathrm{~min}$ at the same temperature, $6 \mathrm{~mL}$ of polyethylene glycol (PEG) was added to the resulting solution. The polymerization was performed for $24 \mathrm{~h}$. After the polymerization, the solution was precipitated in methanol. The precipitate was filtered, washed with hot water, and dried at $30^{\circ} \mathrm{C}$ in a vacuum oven. The percentage graft yield $(G \%)$ was calculated gravimetrically. ${ }^{15,20}$

\subsection{Blend membrane preparation}

The casting solutions were formulated by dissolving AMC and CA- $g$-(GMA- $g$-PEG) in various concentrations using NMP as the solvent, and the solution was kept in an Erlenmeyer flask capped by hollow plugs and wrapped with Teflon tape to prevent evaporation of the solvent. The solution was homogenized using a rotator (RT-10, Wise Mixs, Korea). As shown in Table 1, a series of blend solutions were prepared by varying the composition of AMC and CA-g-GMA-g-PEG. NWF was used as the support layer. The membranes were cast on to this substrate using an adjustable thickness casting knife (YBA-5, Baker applicator, Yoshimitsu, Japan) at a constant temperature (25 \pm $2{ }^{\circ} \mathrm{C}$ ) and relative humidity (40\%) chamber. After casting, the solvent present in the cast film was allowed to evaporate for $30 \mathrm{~s}$ and the cast film along with the glass plate was gently immersed in a coagulation bath consisting of $2.0 \%(\mathrm{v} / \mathrm{v}) \mathrm{NMP}$ (solvent) and $0.2 \mathrm{wt} \%$ SLS. After $2 \mathrm{~h}$ of gelation, the membranes were removed from the coagulation bath and washed thoroughly with distilled water to remove all the NMP and surfactant from the membranes. The membrane sheets were subsequently stored in distilled water prior to performance testing.

\subsection{Ultrafiltration experimental setup}

The UF experiments were performed using a cross-flow filtration setup in which two membrane cells were connected in

Table 1 Composition and casting conditions of the AMC/CA-g(GMA-g-PEG) blend membranes ${ }^{a, b}$

\begin{tabular}{lll}
\hline \multicolumn{2}{l}{ Blend composition (w/w) } & \\
\cline { 1 - 2 } AMC (wt\%) & CA- $g$-(GMA- $g$-PEG) (wt $\%)$ & Solvent, NMP (wt\%) \\
\hline 100 & 0 & 86 \\
90 & 10 & 86 \\
80 & 20 & 86 \\
70 & 30 & 86
\end{tabular}

${ }^{a}$ Casting temperature $=25 \pm 1{ }^{\circ} \mathrm{C}$; casting relative humidity $=40 \pm 2 \%$; casting thickness $=150 \mu \mathrm{m}$; solvent evaporation time $=30 \mathrm{~s}^{b}{ }^{b}$ Total polymer concentration at $14 \mathrm{wt} \%$. 
series. The cross-flow cell houses flat sheet circular membrane pieces had an effective area of $19.6 \mathrm{~cm}^{2}$. The feed solution was passed through the membrane surface at a flow rate of $1.6 \mathrm{~L}$ $\min ^{-1}$. The valve located at the end of the cell was used to pressurize the feed solutions and control the feed pressure.

\subsection{Characterization}

2.5.1 ${ }^{1}$ H-NMR, X-ray photoelectron spectroscopy (XPS), $\mathrm{X}$-ray diffraction (XRD) and thermo gravimetric analysis (TGA). ${ }^{1} \mathrm{H}-\mathrm{NMR}$ spectra of the pure CA- $g$-(GMA- $g$-PEG) powder were obtained using an AM-400 spectrometer (Bruker, USA) operating at a frequency of $400 \mathrm{MHz}$. Spectra were acquired at room temperature using deuterated chloroform solvent and referenced to an internal tetramethylsilane standard. ${ }^{1} \mathrm{H}-\mathrm{NMR}$ spectra were collected at a spectral width of 4000 and $20000 \mathrm{~Hz}$.

XPS of the pure CA and CA- $g$-(GMA- $g$-PEG) powder was conducted using a Thermo Fisher, UK spectrometer. The radiation source was monochromatic $\mathrm{Al} \mathrm{K \alpha}$ operating at a power of $300 \mathrm{~W}$ (15 kV, $20 \mathrm{~mA})$.

Wide angle X-ray diffraction (WAXD) patterns of the pure AMC, AMC/CA- $g$-(GMA- $g$-PEG) blend membranes and CA- $g$ (GMA- $g$-PEG) powder were recorded using a Bruker AXS D8 Advance X-ray diffractometer. The diffractograms were measured at the diffraction angles $(2 \theta)$ in the range $5-50^{\circ}$ using $\mathrm{Cu} \mathrm{K} \alpha$ radiation $(\lambda=1.5406 \AA)$ and a tube voltage of $40 \mathrm{kV}$. The tube current of $30 \mathrm{~mA}$ was kept constant throughout the experiment.

The thermal stability of the pure CA- $g$-GMA- $g$-PEG powder and AMC blend membranes was evaluated using a Universal V4.5A TA DTG analyzer in nitrogen atmosphere. The samples were heated from room temperature to $800{ }^{\circ} \mathrm{C}$ at a heating rate of $10{ }^{\circ} \mathrm{C} \min ^{-1}$.

2.5.2 Microscopy analysis. The cross-sectional images of the membranes were observed by field emission scanning electron microscopy (FESEM Nano230, FEI, USA). Each membrane sample was dried at $30{ }^{\circ} \mathrm{C}$ for $24 \mathrm{~h}$ in an oven to dehydrate and then rigidly mounted on a specimen using conductive adhesive carbon tape. The cross-section of the sample was obtained by breaking the membrane in liquid nitrogen. To reduce image artifacts caused by the electrostatic charge, all the samples were Pt-coated at $20 \mathrm{~mA}$ and $2 \times 10^{-3}$ mbar for $60 \mathrm{~s}$ using a Turbo Pumped High-Resolution Chromium Sputter Coater (K575X, EMITECH, Germany).

The surface morphologies of the pure AMC and AMC/CA- $g$ (GMA- $g$-PEG) blend membranes were examined using a Multimode V (Veeco, USA) atomic force microscope (AFM) capable of imaging at vertical lateral resolutions of $0.1 \AA$. The samples were measured in the tapping mode using a cantilever tip oscillating at its resonant frequency. The changes in the oscillation amplitude of the cantilever because of the interactions between the sample and the cantilever tip were recorded and the topography of each sample was obtained by mapping the corresponding deviations from the target amplitude in voltage. Small squares of the prepared membranes $\left(\sim 1 \mathrm{~cm}^{2}\right)$ were cut and glued onto a substrate. The membrane surfaces were imaged in a scan size of $1 \times 1 \mu \mathrm{m}^{2}$.
CLSM was used to analyze the surface morphology and roughness of the fouled AMC blend membranes (CLSM, OLS2000, Olympus, Japan). A laser beam passes through a light source aperture and is then focused by an objective lens into a small focal volume within a fluorescent specimen. A beam splitter separates the light mixture by allowing only the laser light to pass through reflecting the fluorescent light into the detection apparatus. After passing through a pinhole, the fluorescent light is detected using a photo-detector. The membrane surfaces were imaged in a scan size of $10 \times$ and the surface roughnesses were measured.

2.5.3 Measurement of contact angle and surface free energy. Water contact angles on the membrane surfaces were measured by the sessile drop method at ambient temperature using a Goniometer (Phoenix300 Plus, Surface \& Electro Optics Co. Ltd., Korea). The membrane samples were dehydrated at $50{ }^{\circ} \mathrm{C}$ for $24 \mathrm{~h}$ in an oven before the measurement. Two replicates were used and five liquid drops per replicate were placed on the membranes for the average value of the contact angle. From the measured contact angle values the surface free energy parameters of the membranes were calculated using the Neumann method. The wetting energy, work of adhesion $\left(W_{\mathrm{a}}\right)$ and spreading coefficient $\left(S_{\mathrm{c}}\right)$ were calculated using eqn (1)-(3) $)^{\mathbf{2 1 , 2 2}}$

$$
\begin{gathered}
r_{\mathrm{sl}}=r_{\mathrm{sv}}-r_{\mathrm{lv}} \cos \theta_{\gamma} \\
W_{\mathrm{a}}=r_{\mathrm{s}}^{\circ}+r_{\mathrm{lv}}^{\circ}-r_{\mathrm{sl}} \\
S_{\mathrm{c}}=r_{\mathrm{sv}}-r_{\mathrm{sl}}-r_{\mathrm{lv}}
\end{gathered}
$$

$r_{\mathrm{lv}}$ is the liquid-vapour, $r_{\mathrm{sv}}$ the solid-vapour, and $r_{\mathrm{sl}}$ the solidliquid interfacial free energies respectively and $\theta$ is the measured angle with respect to the surface.

2.5.4 Equilibrium water content, porosity and pore size. The equilibrium water content of the membranes was obtained by soaking the membranes in water for $24 \mathrm{~h}$ and weighing them after mopping with blotting paper. These wet membranes were placed in a vacuum oven at $35^{\circ} \mathrm{C}$ for $48 \mathrm{~h}$ and the dry weights were determined. From these two values, the water content was derived as follows: ${ }^{23}$

$$
\text { Water content }(\%)=\frac{m_{1}-m_{2}}{m_{1}} \times 100
$$

where $m_{1}$ is the weight of the wet membrane and $m_{2}$ is the weight of the dry membrane.

The membrane porosity $(\varepsilon)$ was determined by the gravimetric method by eqn $(5)^{\mathbf{2 4 , 2 5}}$

$$
\varepsilon=\frac{m_{1}-m_{2}}{\rho_{\mathrm{w}} A l}
$$

where $\rho_{\mathrm{w}}$ is the water density $\left(0.998 \mathrm{~g} \mathrm{~cm}^{-3}\right), A$ is the effective area of the membrane $\left(\mathrm{cm}^{2}\right)$, and $l$ is the membrane thickness (cm).

The molecular weight has a linear relationship with the pore size of the membrane. In general, the MWCO of the membrane is determined by identifying an inert of lowest molecular weight that have a solute rejection of $80-90 \%$ in steady-state UF experiments. The molecular weight $(M)$ of the used PEGs were 
correlated with their Stokes-Einstein radii and this enables to calculate the average pore radius $(R)$ of the membranes. ${ }^{26}$

$$
R=\left(16.73 \times 10^{-10}\right) M^{0.557}
$$

2.5.5 Pure water flux (PWF) and organic rejection. All the membranes were compacted at 60 psi transmembrane pressure, whereas the pure water flux, rejection and fouling studies were performed at a transmembrane pressure of $50 \mathrm{psi}$. PWF was measured after a stabilization period of $30 \mathrm{~min}$, and calculated under the steady state conditions using eqn (7). PWF was measured thrice and the average was reported for accuracy.

$$
J_{\mathrm{w}}=\frac{Q}{A(\Delta T)}
$$

$J_{\mathrm{w}}$ is the water flux $\left(\mathrm{L} \mathrm{m}^{-2} \mathrm{~h}^{-1}\right), Q$ is the quantity of the permeate collected (L), $\Delta T$ is the sampling time (h) and $A$ is the membrane area $\left(\mathrm{m}^{2}\right)$.

BSA, SA, and HA stock solutions (15 ppm) were prepared by dissolving the foulant in DI water $(\mathrm{pH}=7)$ and used as natural organic matter (NOM) standard solutions for the rejection studies. Filtration through each membrane was carried out individually, and the concentration of the feed solution was kept constant throughout the run. The rejection was estimated using a total organic carbon analyzer (Shimadzu, TOC-V CPH). The percentage of the foulant rejection was calculated from the concentration of the feed and permeate using eqn (8).

$$
\operatorname{Rejection}(\%)=\left[1-\left(\frac{C_{\mathrm{p}}}{C_{\mathrm{f}}}\right)\right] \times 100
$$

where $C_{\mathrm{f}}$ and $C_{\mathrm{p}}$ are the concentrations of the foulant in the feed and permeate solutions respectively.

2.5.6 Antifouling performance of AMC blend membranes. For the fouling studies, aqueous BSA, HA and SA feed solutions were prepared at concentrations of $15 \mathrm{ppm}$ each (pH 7.0). Each membrane was initially compacted for $15 \mathrm{~min}$, and the PWF, $J_{\mathrm{w} 1}$ was measured at a transmembrane pressure of $50 \mathrm{psi}$, according to eqn (7). The cell was then emptied and refilled with the feed solution. The steady state foulant solution flux, $\left(J_{\mathrm{p}}\right)$ was recorded after $3 \mathrm{~h}$ of filtration. To evaluate the antifouling property of the membranes, the flux decline rate $\left(R_{\mathrm{fd}}\right)$ was also calculated. ${ }^{27}$

When the substance is retained on the membranes and/or blocks the membrane pores, the substance contributes resistance to the flow. Therefore, the extent of the membrane fouling can be calculated from the resistance in the series model using eqn $(9)^{28}$

$$
\frac{\mathrm{d}(v)}{\mathrm{d} t}=J_{\mathrm{w}}=\frac{\Delta p}{\mu\left(R_{\mathrm{m}}+R_{\mathrm{p}}+R_{\mathrm{c}}\right)}
$$

where $R_{\mathrm{m}}\left(\mathrm{m}^{-1}\right)$ is the membrane hydraulic resistance, $R_{\mathrm{p}}\left(\mathrm{m}^{-1}\right)$ is the resistance due to pore blocking, and $R_{\mathrm{c}}\left(\mathrm{m}^{-1}\right)$ is the resistance arising from cake formation..$^{29,30}$

The degree of irreversible flux loss caused by irreversible fouling $\left(R_{\mathrm{ir}}\right)$ and reversible flux loss caused by reversible fouling $\left(R_{\mathrm{r}}\right)$ were calculated using eqn (10) and (11), respectively.

$$
\begin{gathered}
R_{\mathrm{ir}}=\left[\frac{J_{\mathrm{w} 1}-J_{\mathrm{w} 2}}{J_{\mathrm{w} 1}}\right] \times 100 \\
R_{\mathrm{r}}=\left[\frac{J_{\mathrm{w} 2}-J_{\mathrm{p}}}{J_{\mathrm{w} 1}}\right] \times 100
\end{gathered}
$$

$J_{\mathrm{w} 1}$ is the PWF before foulant solution filtration, $J_{\mathrm{w} 2}$ is the PWF after cleaning, $\mu$ is the viscosity of water and $J_{\mathrm{p}}$ is the flux with the filtration of foulant solutions.

To evaluate the antifouling property of AMC/CA- $g$-(GMA- $g$ PEG) blend membranes, the flux recovery ratio (FRR) was calculated using eqn (12):

$$
\operatorname{FRR}(\%)=\left[\frac{J_{\mathrm{w} 2}}{J_{\mathrm{w} 1}}\right] \times 100
$$

\section{Results and discussion}

\subsection{Synthesis of CA- $g$-(GMA- $g$-PEG) polymer}

The ${ }^{1} \mathrm{H}$-NMR spectrum of the prepared polymer shown in Fig. 1 illustrates the successful synthesis of CA-g-(GMA-g-PEG). The region at 4.5-5.0 ppm confirms the $\mathrm{CH}$ protons of the cellulose acetate backbone. The peak in the range $2.7-2.8 \mathrm{ppm}$ is assigned to the substituted epoxy hydrogen of GMA. The peak at $2.0 \mathrm{ppm}$ shows the end hydroxyl protons of PEG and the singlet at $3.7 \mathrm{ppm}$ confirms the $-\mathrm{OCH}_{2}$ - protons of $\mathrm{PEG}$, indicating the bond breakage in epoxy ring and the PEG is attached to the epoxy ring of GMA. The above results confirm (GMA-g-PEG) grafting onto the cellulose acetate. The grafting yield calculated was found to be $40.3 \%$.

X-ray photoelectron spectra were obtained to evaluate the chemical composition of the polymers. High resolution C1s XPS spectra, C1 binding energies and atomic concentration percentages of pure CA and grafted CA are shown in Fig. 2. The XPS spectra of the unmodified and modified CA show that carbon and oxygen atoms are the main components. Pure cellulose acetate showed three distinct carbon peaks - the peak at $286.78 \mathrm{eV}$ corresponding to the carbon atoms in the $\mathrm{C}-\mathrm{O}-\mathrm{H}$ group, the peak at $289.1 \mathrm{eV}$ corresponding to the carbons in $\mathrm{O}-\mathrm{C}=\mathrm{O}$ and $\mathrm{O}-\mathrm{C}-\mathrm{O}$ and the peak of $285.2 \mathrm{eV}$ representing the reference carbon of the hydrocarbon and $-\mathrm{CH}_{2}-$ carbons of PEG or cellulosic polymer. However, the high resolution C1s spectra of the modified CA showed almost a total overlap of the peaks corresponding to the carbon moieties which had the lower binding energies $(286.78$ and $285.2 \mathrm{eV}$ ). This can be explained by the decrease in the peak intensity of the carbon attributed to the $\mathrm{C}-\mathrm{O}-\mathrm{H}$ group, and an increase in the peak intensity corresponding to the hydrocarbon and $-\mathrm{CH}_{2}-$ carbons. Furthermore, the atomic percentage of carbon for the grafted CA is higher than that of the pure CA. In contrast the atomic percentage of oxygen is lower for the modified CA. The overall result confirms the grafting of GMA and PEG monomers onto the cellulose backbone.

\subsection{XRD analysis}

The crystalline/amorphous structure of binary blend composite membrane is essential to examine the mechanism of selectivity 


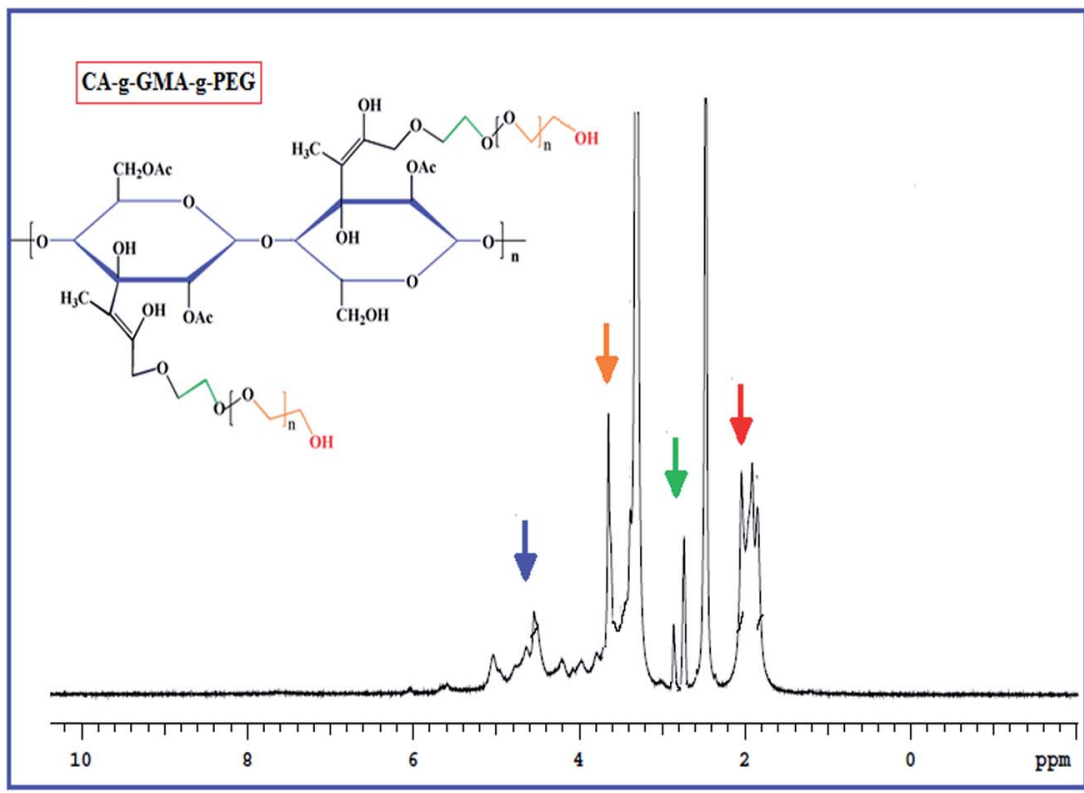

Fig. $1{ }^{1} \mathrm{H}$ NMR spectrum of CA-g-GMA- $g$-PEG.

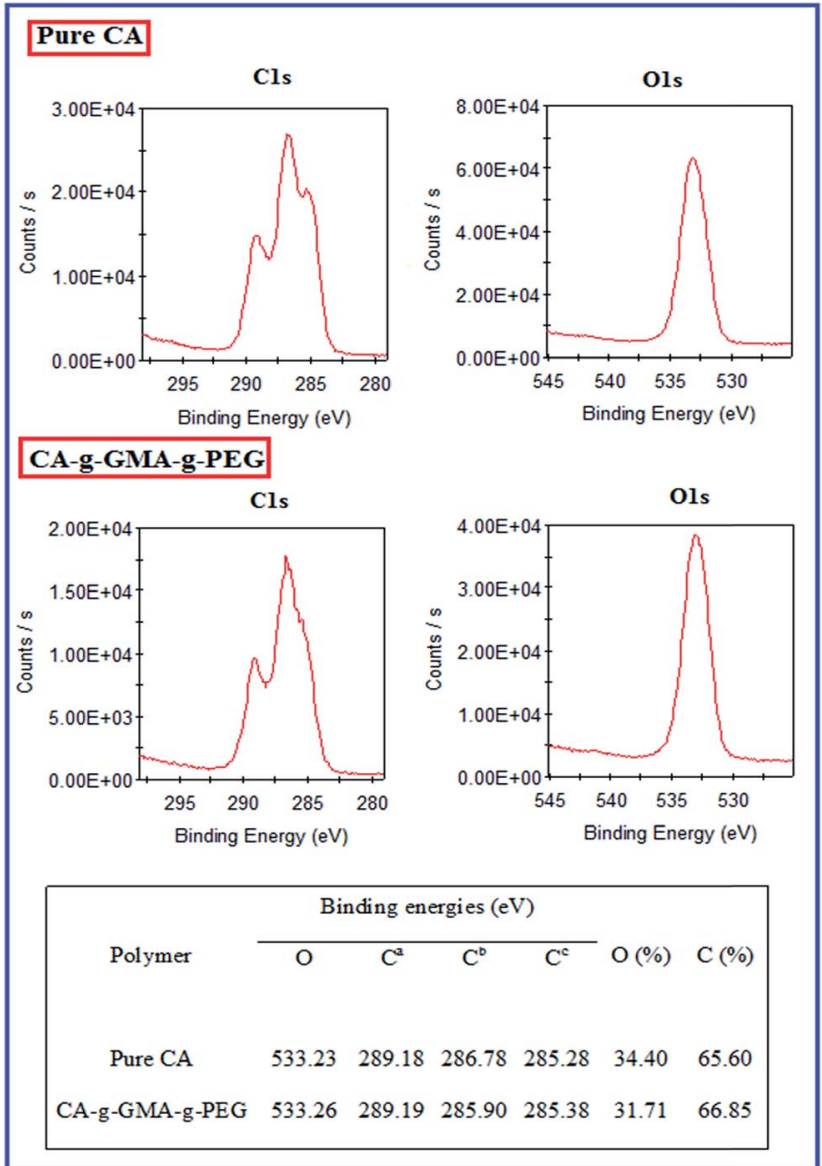

Fig. $2 \mathrm{C} 1 \mathrm{~s}$ and O1s XPS peaks, binding energies and atomic percentages of pure CA and CA-g-GMA-g-PEG. and permeability during the UF process. The crystalline structure of pure AMC membrane, the pure CA- $g$-(GMA- $g$-PEG) powder, and AMC blend membranes were probed with XRD and the results are shown in Fig. 3. The diffraction pattern of pure AMC membrane shows a broad semi crystalline peak at $18.4^{\circ}$ and two sharp crystalline peaks at $22.7^{\circ}$ and $25.8^{\circ}$. The CA- $g$ (GMA-g-PEG) powder showed a broad diffraction peak at $19.7^{\circ}$ signifying its amorphous structure and a semi crystalline diffraction peak at $26.5^{\circ}$. The AMC blend membranes showed the diffraction peaks of both the AMC and grafted CA. The most notable feature of the AMC blend membranes is the enhancement of the amorphous structure owing to the incorporation of CA- $g$-GMA- $g$-PEG. The low intensity of all the diffraction peaks confirms the amorphous nature of the AMC blend membranes. The crystalline phase of the polymer chains is owing to their tight packing, affecting the material transport. ${ }^{31}$ However, the polymer chains in the amorphous phase are loosely packed, easing the material transport.

\subsection{TGA analysis}

The thermal stability of the pure CA- $g$-(GMA- $g$-PEG) powder, and AMC blend membranes are shown in Fig. 4. The initial degradation temperature $\left(T_{\text {on }}\right)$ and maximum weight loss $\left(T_{\max }\right)$ at different temperature have been analyzed. From the resultant thermograms, pure CA- $g$-(GMA- $g$-PEG) shows better thermal stability than AMC membranes. The weight loss temperature is decreased slightly due to the incorporation of grafted CA in the AMC dope solution. The initial degradation temperature of grafted CA and AMC membrane is obtained at $362{ }^{\circ} \mathrm{C}$ and 338 ${ }^{\circ} \mathrm{C}$. The maximum weight loss in pure CA- $g$-(GMA- $g$-PEG) observed at $479{ }^{\circ} \mathrm{C}$, while AMC membranes showed a temperature at $472{ }^{\circ} \mathrm{C}$. The maximum temperature values at $460{ }^{\circ} \mathrm{C}$, $448{ }^{\circ} \mathrm{C}$ and $412{ }^{\circ} \mathrm{C}$ for the concentration of grafted CA $10 \%, 20 \%$ 


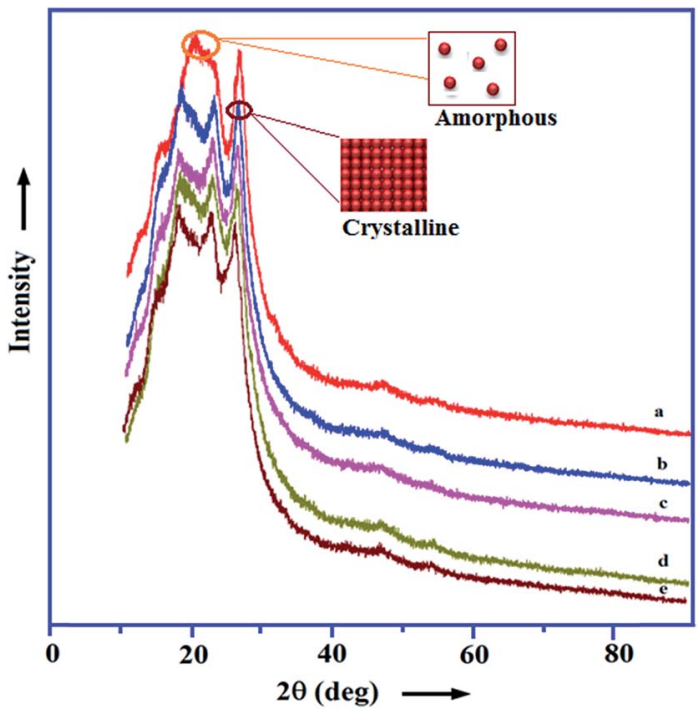

Fig. 3 XRD patterns of the AMC/CA-g-(GMA-g-PEG) blend membranes and pure CA-g-(GMA-g-PEG) powder: (a) pure CA-gGMA-g-PEG; (b) 100/0; (c) 90/10; (d) 80/20; (e) 70/30.

and $30 \%$ respectively. The change in thermal behaviour for pure grafted CA and AMC bend membranes was confirmed by TGA analysis. As found in literature, the temperature range of thermal degradation of AMC from $325{ }^{\circ} \mathrm{C}$ to $415{ }^{\circ} \mathrm{C}$ that interprets the loss of acetic acid and oxides of carbon. ${ }^{32}$

\subsection{Microscopic analysis of the AMC blend membranes}

The cross-sectional SEM images of the AMC blend membranes prepared at various polymer compositions are shown in Fig. 5 . Typically, the asymmetric structure of the cellulose-based membrane has a dense skin layer and a thick porous sub layer representing two types of macrovoids - finger-like and

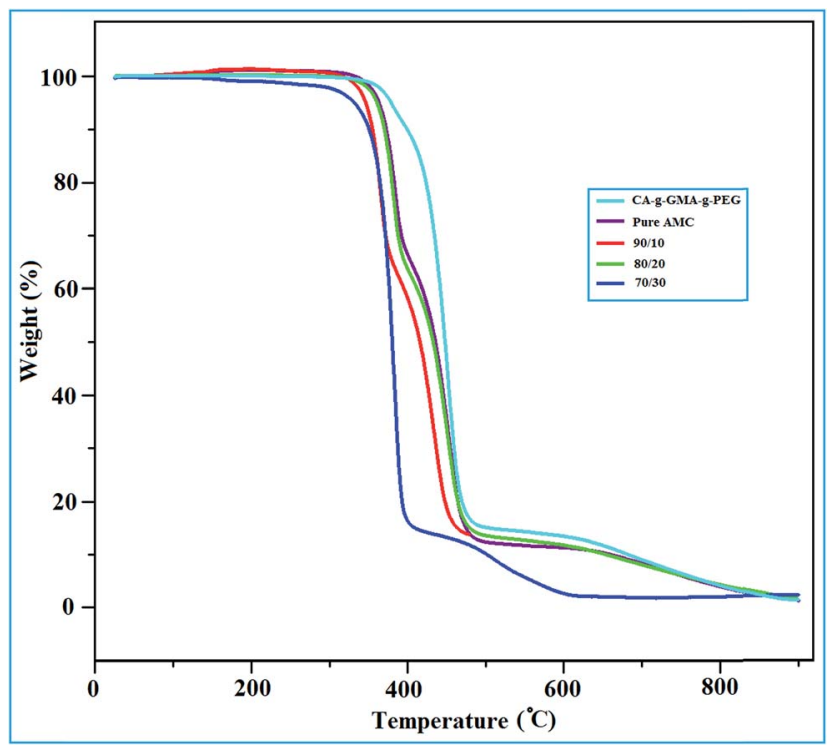

Fig. 4 TGA patterns of the pure CA-g-(GMA-g-PEG) powder and AMC/CA-g-(GMA-g-PEG) blend membranes. irregular (such as tear-like, and channel-like) structures. ${ }^{33}$ The micrographs of the pure AMC membranes exhibited irregular macrovoids over the entire membrane cross section in contrast to the sponge like structure of the AMC/CA- $g$-(GMA- $g$-PEG) blend membranes. Moreover, the pore size of the AMC membranes decreased with increasing portion of the grafted CA polymer. These changes in the morphology of the AMC blend membranes revealed the decisive role of the grafted CA polymer in the structural growth during the membrane preparation. The porous structure of the AMC blend membranes has a positive effect in improving the mechanism of the permeability and selectivity.

The surface structure of the membrane prepared by the immersion precipitation is regulated by the mutual diffusion of both the solvent (NMP) and non-solvent (water) into the sub layers of the dope solution. The rate of inward diffusion of water is high compared to the outward diffusion of NMP because of the slight diffusion of water molecules compared to those of the bulkier organic solvent molecules. ${ }^{34}$ However, in this case, the diffusion of water into the casting solution was slowed down by the presence of the hydrophilic grafted polymer in the AMC matrix. This delayed de-mixing in the AMC/CA- $g$-(GMA-g-PEG) blend membranes favors the nucleation, forming the large percentages of the polymer poor phase, thus resulting in a high porosity in the sub layer of the membrane. The number of pores present in the sub layer is higher in the AMC blend membranes
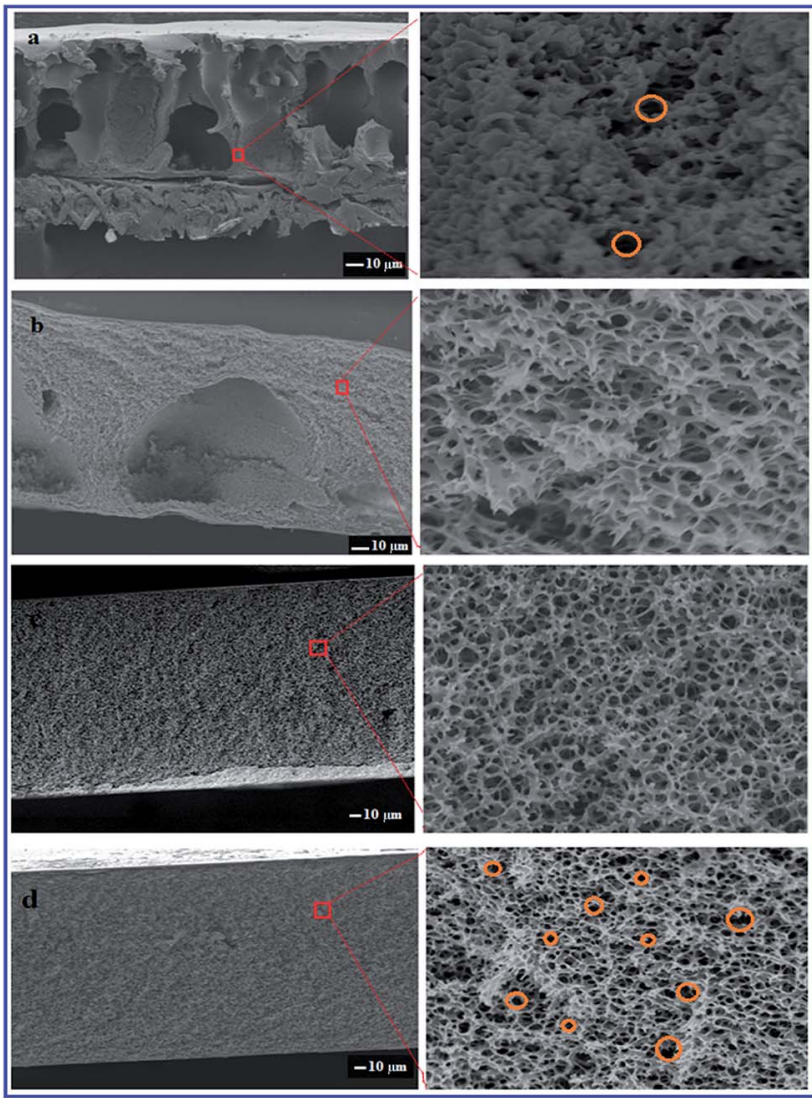

Fig. 5 Cross sectional SEM images of AMC/CA- $g$-(GMA- $g$-PEG) blend membranes (w/w): (a) 100/0; (b) 90/10; (c) 80/20; (d) 70/30. 
Table 2 AFM surface roughness, pure water flux, water content, pore size, and porosity of AMC/CA-g-(GMA-g-PEG) blend membranes

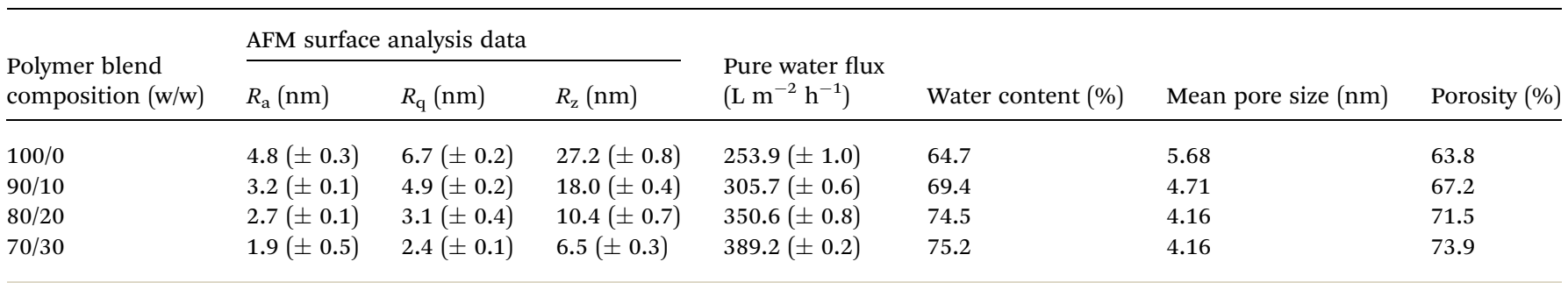

when compared to the pure AMC membranes (marked in yellow color in SEM images), clearly indicating that the pores are not opened properly in the pure AMC due to the faster de-mixing.

The influence of CA-g-(GMA- $g$-PEG) on both the membrane morphology and surface roughness were studied by the AFM analysis. Fig. 6 represents the three dimensional AFM images of pure AMC and AMC/CA- $g$-(GMA- $g$-PEG) blend membranes. In the AFM images, the surfaces of both unmodified and modified membranes demonstrate a nodule-valley like structure. The morphology of the blend membranes altered with the addition of the grafted CA in the casting solution and the surface properties of the AMC membranes significantly improved, resulting in a smoother surface at a blend composition of 70/30. In the case of smooth membranes, even if the same number of particles were deposited, they were likely to be more uniformly spaced resulting in a low flux decline. Thus, at the smooth membrane surface, no flux decline was reported until a dense cake layer developed. ${ }^{35}$ The pure AMC membrane surface showed large nodules, and with increasing the grafted CA content, the nodules decreased their size signifying a minimization in pore size of the blend membranes (marked in blue color in the AFM images).

The three important surface roughness parameters $\left(R_{\mathrm{a}}-\right.$ the mean value of the surface relative to the center plane, $R_{\mathrm{q}}-$ the root mean square of the $Z$ data and $R_{\mathrm{z}}$ - the mean difference between the highest peaks and lowest valleys) were evaluated from the AFM images and the results are reported in Table 2. The surface roughness of the modified AMC membranes decreased when compared to the virgin AMC membranes. The surface roughness of the membranes is correlated to the colloidal fouling, confirming that with decreasing surface roughness, colloidal fouling decreases. ${ }^{36}$ Many studies indicate that the change in the roughness parameters of the polymeric membranes is proportional to the change in the pore size..$^{37,38}$

\subsection{Contact angle analysis}

The wetting behaviors of liquids on the membrane surfaces were examined by measuring the contact angles, and the surface energy parameters were calculated for the membranes.

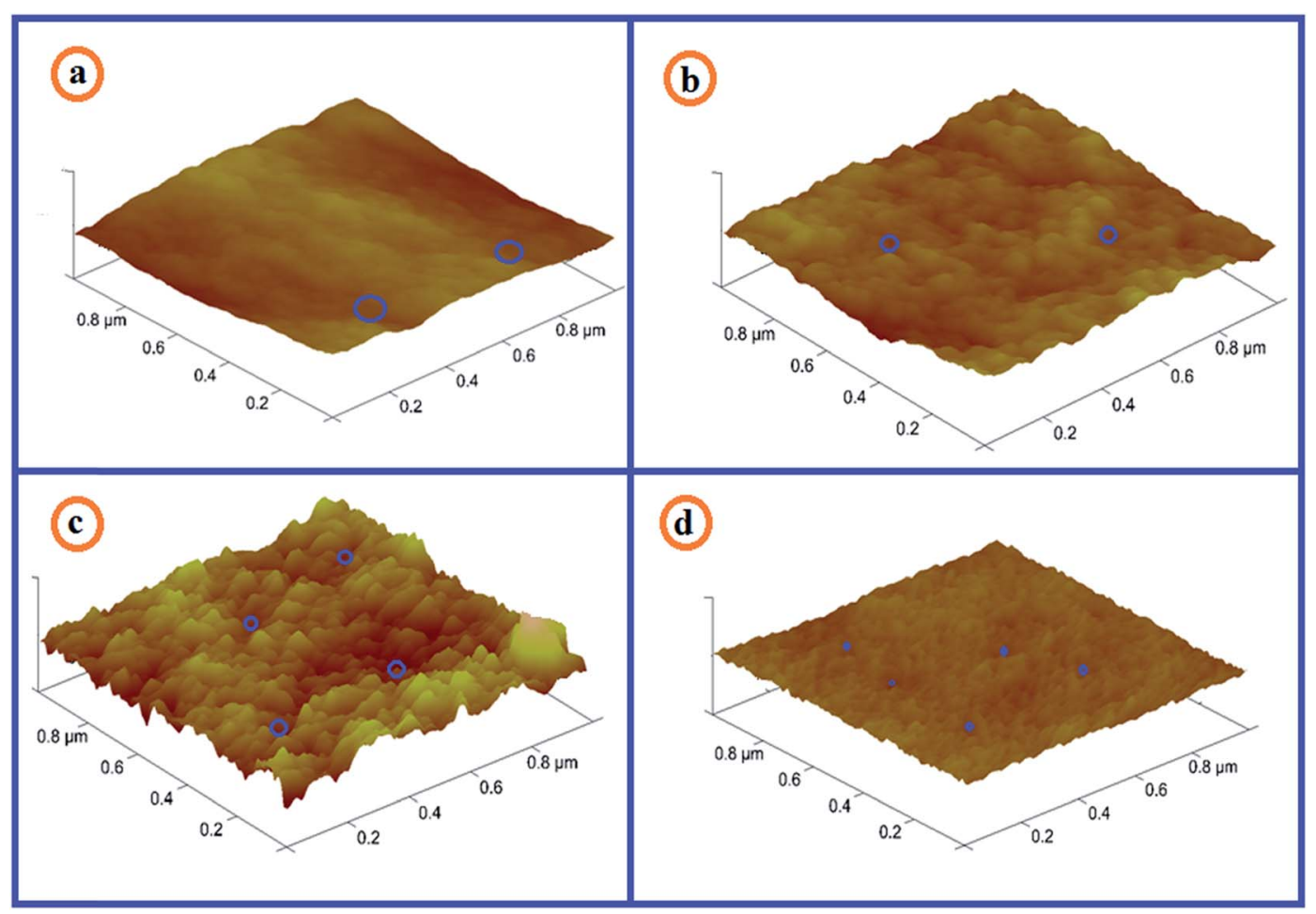

Fig. 6 Three dimensional AFM images of AMC/CA-g-(GMA-g-PEG) blend membranes (w/w): (a) 100/0; (b) 90/10; (c) 80/20; (d) 70/30. 
The measured contact angles presented in Table 3 show that the hydrophilicity of the blend membranes is higher than those of the pure AMC membranes. The incorporation of CA- $g$-GMC- $g$ PEG enhanced the wettability of the membranes. The contact angle measurement taken at thermodynamic equilibrium of a polymeric membrane measures its wettability and is affected by the chemical composition of the membrane surface, porosity, and roughness. The potentiality of individual atoms and substituent groups in increasing the wetting properties of the membranes is in the following order: $\mathrm{N}>\mathrm{O}>\mathrm{I}>\mathrm{Cl}>\mathrm{H}>\mathrm{F}^{39}$ The AMC/CA- $g$-GMC- $g$-PEG blend membranes show lower contact angles due to the presence of hydrogen bonding and van der Waals interactions in the grafted CA.

From the measured contact angle values, various surface parameters such as wetting energy, work of adhesion, and spreading coefficient were calculated, and the results are listed in Table 3. Wetting energy increased for the blend membranes, indicating the enhanced hydrophilicity of the membranes. In most membrane processes, particularly in bio-separations, higher wetting energy surfaces correspond to lower fouling. The $W_{\mathrm{a}}$ follows the same trend as the wetting energy, i.e., the $W_{\mathrm{a}}$ values increased with increasing CA- $g$-(GMA- $g$-PEG) composition. This leads us to conclude that the surface of the AMC membrane obtained by blending with CA- $g$-(GMA- $g$-PEG) was sufficient to increase its adhesive properties. The spreading coefficient values obtained reveal that as the CA- $g$-(GMA- $g$-PEG) composition increased in the blend system, the spreading coefficient decreased, thereby increasing the wettability.

\subsection{Pure water flux (PWF), water content, pore size and porosity}

The pure water flux of AMC/CA- $g$-(GMA- $g$-PEG) blend membranes was measured after an initial stabilization period of $30 \mathrm{~min}$, and the results are shown in Table 2. The PWF of the membrane increased with increasing concentration of CA- $g$ (GMA- $g$-PEG) in the casting solution and reached a maximum value of $389.2 \mathrm{~L} \mathrm{~m}^{-2} \mathrm{~h}^{-1}$ at a blend composition of 70/30. The membrane permeability of the AMC blend membranes significantly improved, and the membrane pores were clearly observed by the SEM analysis. The addition of CA- $g$-(GMA- $g$ PEG) to the dope solution resulted in a higher number of pores in the membrane layer in comparison to the pure AMC membranes and the porous membrane layer encourages the water transport through the membranes.

Table 3 Wetting energy, $W_{a}$, and $S_{c}$ values calculated from the contact angle values

\begin{tabular}{lllll}
\hline $\begin{array}{l}\text { Polymer blend } \\
\text { composition } \\
(\mathrm{w} / \mathrm{w})\end{array}$ & $\begin{array}{l}\text { Contact } \\
\text { angle }(\theta)\end{array}$ & $\begin{array}{l}\text { Wetting } \\
\text { energy, } \\
\left(\mathrm{mN} \mathrm{m}^{-1}\right)\end{array}$ & $\begin{array}{l}\text { Work of } \\
\text { adhesion, } \\
\left(\mathrm{mN} \mathrm{m}^{-1}\right)\end{array}$ & $\begin{array}{l}\text { Spreading } \\
\text { coefficient, } \\
\left(\mathrm{mN} \mathrm{m}^{-1}\right)\end{array}$ \\
\hline $100 / 0$ & $53.2( \pm 1.2)$ & 43.5 & 116.3 & 29.2 \\
$90 / 10$ & $46.3( \pm 2.0)$ & 50.2 & 123.0 & 22.5 \\
$80 / 20$ & $42.5( \pm 1.5)$ & 53.6 & 126.4 & 19.1 \\
$70 / 30$ & $40.6( \pm 1.1)$ & 55.2 & 128.0 & 17.5
\end{tabular}

The water content of the membrane is interconnected to the PWF and the wetting property of the membranes. The water content of the pure AMC membrane was found to be $64.7 \%$, as shown in Table 2. In the AMC/CA- $g$-(GMA- $g$-PEG) polymer blend, as the grafted CA content increased, the water content improved noticeably, and at $30 \%$ of CA- $g$-(GMA- $g$-PEG) the water content was found to be $75.2 \%$. The water content values indicate that the pure AMC membranes adsorbed relatively fewer amounts of weakly hydrogen bonded clusters compared to the higher amounts of strongly hydrogen bonded clusters in the AMC/CA- $g$-(GMA- $g$-PEG) blend membranes. ${ }^{40}$ The AMC blend membranes possess a sponge-like structure in the entire membrane cross section, acting as a domain of water molecules compared to the pure AMC membranes. The polar groups present in the AMC membrane surfaces from the addition of the grafted CA support the hydrogen bonding interaction with water and forming bulkier water clusters in the blend membranes. ${ }^{40}$ Similar trend was observed in the pore size and porosity of the AMC blend membranes.

\subsection{Rejection studies and fouling analysis of AMC blend membranes}

The rejection of the AMC/CA- $g$-(GMA- $g$-PEG) blend membranes for the individual organic foulants such as HA, SA and BSA in the aqueous solutions is shown in Fig. 7. From these rejection values, it was clearly observed that when the concentration of CA- $g$-(GMA- $g$-PEG) in the blend solution increased, the rejection of the organic foulants increased due to the smaller pore size of the membrane surface as shown in the surface morphological studies. The pure AMC membrane shows $84.7 \%$ rejection of BSA which was lower than those of the AMC blend membranes. The other organic foulants SA and HA had rejections of 75.5 and $73.4 \%$ respectively. The percentage rejections of the organic foulants were in the following order: BSA $>$ SA $>$ HA and can be explained by the molecular weights of the organic foulants. The macromolecular BSA resulted in a rapid blocking of the membrane pores leading to higher rejection of the AMC blend membranes. In the case of HA and SA foulants with lower

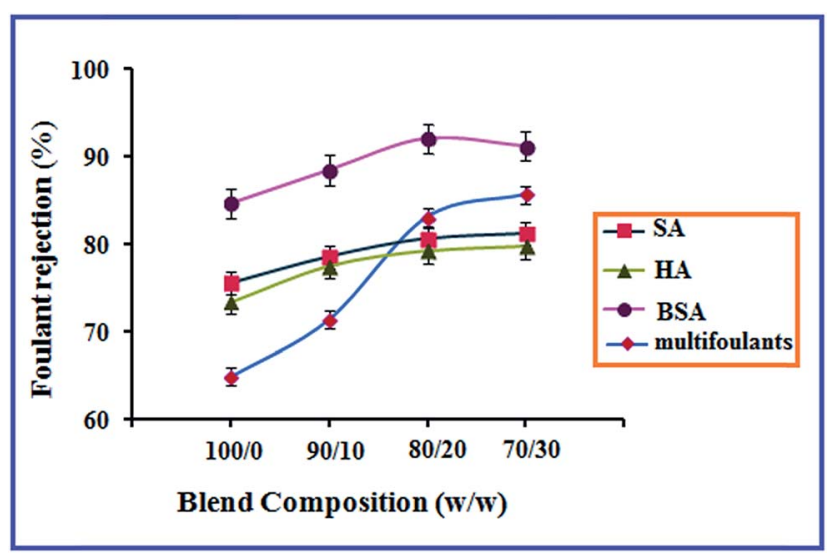

Fig. 7 Rejection of singlet foulant and multi foulants by AMC blend membranes as a function of blend composition. 
Table 4 Summary of the data of flux decline rate, flux recovery ratio, reversible fouling, irreversible fouling, membrane resistance and total resistance of AMC blend membranes

\begin{tabular}{llllllr}
\hline $\begin{array}{l}\text { Polymer blend } \\
\text { composition }(\mathrm{w} / \mathrm{w})\end{array}$ & $\begin{array}{l}\text { Flux decline } \\
\text { rate, } R_{\mathrm{fd}}(\%)\end{array}$ & $\begin{array}{l}\text { Flux recovery } \\
\text { rate, FRR }(\%)\end{array}$ & $\begin{array}{l}\text { Reversible } \\
\text { fouling, } R_{\mathrm{r}}(\%)\end{array}$ & $\begin{array}{l}\text { Irreversible } \\
\text { fouling, } R_{\mathrm{ir}}(\%)\end{array}$ & $\begin{array}{l}\text { Membrane } \\
\text { resistance, } R_{\mathrm{m}} \times 10^{10}\end{array}$ & $\begin{array}{l}\text { Total resistance, } \\
R_{\mathrm{t}} \times 10^{10}\end{array}$ \\
\hline $100 / 0$ & 83.9 & 65.1 & 27.4 & 34.9 & 2.1 & 15.11 \\
$90 / 10$ & 80.5 & 68.5 & 35.9 & 31.5 & 1.8 & 1.3 \\
$80 / 20$ & 77.1 & 70.1 & 44.2 & 29.8 & 1.1 & 5.41 \\
$70 / 30$ & 75.4 & 75.6 & 52.8 & 24.7 & & 5.84
\end{tabular}

molecular weights, lower rejection rates were observed when compared to BSA molecules.

The results of the combined organic foulants rejection of the AMC blend membranes were also investigated. The addition of the grafted CA from 0 to $30 \%$ to the AMC dope solution showed the rejections of $64.9,71.4,83.1$ and $85.7 \%$, respectively. The AMC blend membranes showed higher rejections than the pure AMC membranes. The mixed foulant increases the mass foulant deposition on the pure AMC membranes owing to its hydrophobicity leading to a low rejection when compared to that for the AMC blend membranes. The separating efficiency of the AMC blend membranes improved owing to the formation of dense layers, offering high resistance to the foulants transport across the membranes, and thus resulting in higher foulant rejection.

The antifouling performance of the pure AMC and AMC/CA$g$-(GMA- $g$-PEG) blend membranes was studied using the mutlifoulants - humic acid (HA), sodium alginate (SA) and bovine serum albumin (BSA) - as the model foulants. Table 4 presents the effect of the CA- $g$-(GMA- $g$-PEG) concentration on the fouling resistance of the AMC membranes, demonstrating that the total fouling resistance of the AMC/CA- $g$-(GMA- $g$-PEG) blend membranes decreased from $15.11 \times 10^{10} \mathrm{~m}^{-1}$ to $5.84 \times 10^{10}$ $\mathrm{m}^{-1}$ with increasing CA- $g$-(GMA- $g$-PEG) content. Moreover, the irreversible fouling of the pure AMC membrane is higher than those of the AMC/CA- $g$-(GMA- $g$-PEG) blend membranes and this leads to major flux decline. Consequently, the involvement of multifoulants accumulated on the pure AMC membranes was much higher than those for the grafted CA incorporated membranes. This reveals that the hydrophilic property of the membrane has a significant effect on the fouling resistance of the membranes.

The low interfacial free energy surface defends lesser fouling because of the components presents in the low surface free energy always tend to enhance the surface in order to diminish the free energy. The adhesion values of the AMC blend membranes are increased which possessing adhesion on the low energy surface is highly potent, and high polar surface energy is very low. The low spreading co-efficient values leads an increase in hydrophilicity of the membrane surface. Thus, the enhancement of low surface free energy and low adhesive surface is the superior alternative for antifouling. ${ }^{41,42}$

With the incorporation of the grafted polymer and the corresponding increase in the hydrophilicity of the AMC membrane surface, the accumulated multifoulants on the
Table 5 CLSM surface roughness of the fouled AMC blend membranes

\begin{tabular}{llll}
\hline & \multicolumn{3}{l}{ Roughness parameters } \\
\cline { 2 - 4 } $\begin{array}{l}\text { Polymer blend } \\
\text { composition }(14 \mathrm{wt} \%)\end{array}$ & $\mathrm{SR}_{\mathrm{a}}(\mathrm{nm})$ & $\mathrm{SR}_{\mathrm{q}}(\mathrm{nm})$ & $\mathrm{SR}_{\mathrm{z}}(\mathrm{nm})$ \\
\hline $100 / 0$ & $7.8( \pm 0.45)$ & $12.1( \pm 0.19)$ & $34.9( \pm 0.54)$ \\
$90 / 10$ & $6.2( \pm 0.28)$ & $10.2( \pm 0.12)$ & $29.3( \pm 0.91)$ \\
$80 / 20$ & $5.0( \pm 0.15)$ & $6.4( \pm 0.21)$ & $24.0( \pm 0.62)$ \\
$70 / 30$ & $4.1( \pm 0.52)$ & $4.8( \pm 0.11)$ & $20.1( \pm 0.30)$
\end{tabular}

membranes can be more readily dislodged by shear force compared to those for the pure AMC membranes. Further, with the high surface roughness of the AMC blend membranes, organic foulants are expected to preferentially migrate into the membrane pores leading to severe pore blockage. On the contrary, the membrane fouling of the AMC blend membranes was largely endorsed to reversible membrane fouling, which can be easily removed by simple water washing as evidenced by the flux recovery data of these membranes. The FRR value of the AMC/CA- $g$-(GMA- $g$-PEG) blend membranes is $75.6 \%$ at $70 / 30$ composition, and this indicates the structure of cake layer, weakly attached to the membrane surface, show a good competence of the hydraulic cleaning with higher antifouling property of the membranes.

\subsection{CLSM of fouled membranes}

CLSM facilitates the study of the external adsorption on the membrane surfaces to certain depths and surface roughness parameters of the membranes determining the significance of fouling. ${ }^{39}$ Hence, to scrutinize the effect of CA- $g$-(GMA- $g$-PEG) on the AMC blend membranes surface and surface roughness, CLSM analysis was performed in the fluorescence mode.

Fig. 8 shows the three dimensional CLSM images of the fouled surface topography of the pure AMC and AMC/CA- $g$ (GMA-g-PEG) blend membranes. The constitution of the accumulated organic foulants layer on the pure AMC and AMC blend membranes are divergent in shape and size representing different extents of fouling when compared to the CLSM images. The fouled AMC membrane reveals a cake layer surface which seems to be much more compact and is expected to cause greater flux reduction than the AMC blend membranes as explained in the filtration experiment. Bowen et al. reported that foulants deposit homogeneously on the hydrophilic 

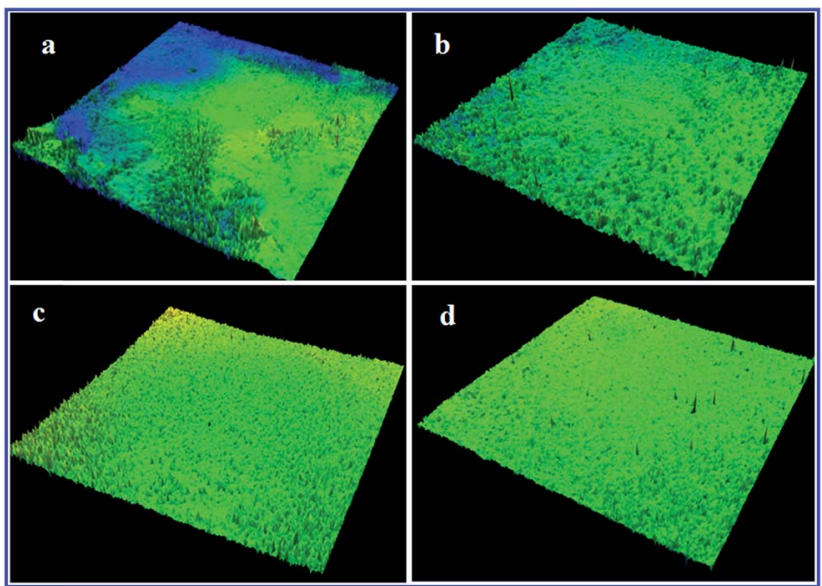

Fig. 8 Three dimensional CLSM images of fouled AMC blend membranes (w/w): (a) 100/0; (b) 90/10; (c) 80/20; (d) 70/30.

surfaces in contrast to the bunch of foulants adsorbed raggedly on the hydrophobic surfaces because of the preferential adsorption of the membrane matrix. ${ }^{43}$

The average surface roughness $\left(\mathrm{SR}_{\mathrm{a}}\right)$ values of the fouled AMC blend membranes with the grafted CA compositions ranging from 0 to $30 \%$ were $7.8,6.2,5.0$, and $4.1 \mathrm{~nm}$, respectively (Table 5 ). The $\mathrm{SR}_{\mathrm{a}}$ values of the fouled membranes are higher than those of the non-fouled membranes (compared to the AFM surface roughness values). The increase in the surface roughness of the fouled membranes may be because of the mass deposition of the macromolecular foulants on the membrane surface. The most fascinating fact is the roughness parameter of the stained membrane well correlated to the formation of the cake layer and the decreased surface roughness because of the more compact gel layer surface of the membrane.

\section{Conclusion}

High performance AMC ultrafiltration membranes were fabricated for diffusion induced phase separation processes using hydrophilic CA grafted copolymer as the modifier. The influence of the CA- $g$-(GMA- $g$-PEG) content on the morphology, permeation properties, hydrophilicity, thermal stability, pore size, porosity, foulant rejection efficiency and fouling propensity of the resultant membranes were evaluated. The synthesis of CA grafted GMA and PEG was confirmed by ${ }^{1} \mathrm{H}-\mathrm{NMR}$, and XPS analyses. The morphology analysis show that increasing the CA- $g$-(GMA- $g$-PEG) content in the AMC matrix reduced the macrovoids and induced sponge like structures in the entire membrane cross section. The surface free energy components of the membranes such as wetting energy, $W_{\mathrm{a}}$, and $S_{\mathrm{c}}$ were calculated from the contact angle and the merit of the study implies the higher hydrophilicity than those of the pure AMC membranes. Therefore, the grafted CA polymer exhibited a positive effect on the membrane performance characterizes as the membrane morphology, porosity, water content, PWF, and surface wettability of the blend AMC membranes. The fouling propensity of the modified AMC membranes decreased when compared to the unmodified AMC membranes because of the higher hydrophilicity and smoother surfaces of the AMC blend membranes. Therefore, CA- $g$-(GMA- $g$-PEG) should be considered as an effective modification agent for the development of low energy, antifouling AMC ultrafiltration membranes for various industrial separations.

\section{Acknowledgements}

This research was supported by the World Premier Materials Program through the Ministry of Trade Industry \& Energy and supported by the R\&D Center for Valuable Recycling (GlobalTop R\&D Program) of the Ministry of Environment [Project no. 2014001160002]. The authors are grateful for support from these organizations.

\section{References}

1 G. M. Geise, H. S. Lee, D. J. Miller, B. D. Freeman, J. E. Mcgrath and D. R. Paul, J. Polym. Sci., Part B: Polym. Phys., 2010, 48, 1685-1718.

2 C. Y. Tang and J. O. Leckie, Environ. Sci. Technol., 2007, 41, 4767-4773.

3 K. Li, H. Liang, F. Qu, S. Shao, H. Yu, Z. S. Han, X. Du and G. Li, J. Membr. Sci., 2014, 471, 94-102.

4 J. Sun, K. Xiao, Y. Mo, P. Liang, Y. Shen, N. Zhu and X. Huang, J. Membr. Sci., 2014, 453, 168-174.

5 Y. Su, C. Mu, C. Li and Z. Jiang, Ind. Eng. Chem. Res., 2009, 48, 3136-3141.

6 A. S. Kim, A. E. Contreras, Q. Li and R. Yuan, Langmuir, 2009, 25, 7815-7827.

7 Y. N. Wang and C. Y. Tang, Environ. Sci. Technol., 2011, 45, 6373-6379.

8 E. Zuriaga-Agusti, E. Alventosa, S. Barredo-Damas, M. I. Alcaina-Miranda, M. I. Iborra-Clar and J. A. MendozaRoca, Water Res., 2014, 54, 199-210.

9 L. Fan, J. L. Harris, F. A. Roddick and N. A. Booker, Water Supply, 2002, 2, 313-320.

10 J. M. Haack, N. A. Booker and T. Carrroll, Water Res., 2003, 37, 585-588.

11 C. Combe, E. Molis, P. Lucas, R. Riley and M. M. Clark, J. Membr. Sci., 1999, 154, 73-87.

12 I. C. Kim, Y. S. Jin, D. H. Song, S. H. Ahn, Y. I. Park, S. Kim, J. Jegal, B. K. Seo, J. H. Kim, Y. N. Kwon, C. J. Mo, J. J. Lee, D. S. Kim and S. H. Lim, Desalin. Water Treat., 2013, 51, 5196-5203.

13 I. C. Kim, S. H. Ahn, Y. S. Jin, B. S. Kim, Y. I. Park, J. H. Kim, J. Jegal, S. H. Lee, Y. N. Kwon and H. W. Rhee, Desalin. Water Treat., 2013, 51, 5191-5195.

14 S. Zhang, K. Y. Wang, T. S. Chung, H. Chen, Y. C. Jean and G. Amy, J. Membr. Sci., 2010, 360, 522-535.

15 A. Jayalakshmi, S. Rajesh, I. C. Kim, S. Senthilkumar, D. Mohan and Y. N. Kwon, J. Membr. Sci., 2014, 465, 117-128.

16 L. Wang, K. Pan, L. Li and B. Cao, Ind. Eng. Chem. Res., 2014, 53, 6401-6408.

17 C. Yagci and U. Yildiz, Eur. Polym. J., 2005, 41, 177-184. 
18 B. Denga, J. Lia, Z. Houa, S. Yaoa, L. Shia, G. Lianga and K. Shenga, Radiat. Phys. Chem., 2008, 77, 898-906.

19 W. Chen, Y. Su, L. Zheng, L. Wang and Z. Jiang, J. Membr. Sci., 2009, 337, 98-105.

20 Z. Yi, L. P. Zhu, Y. F. Zhao, B. K. Zhu and Y. Y. Xu, J. Membr. Sci., 2012, 390-391, 48-57.

21 Z. Xu, X. Huang and L. Wan, Surface engineering of polymer membranes, Springer Berlin Heideilberg, New York, 2009.

22 D. Y. Kwok and A. W. Neumann, Colloids Surf., A, 2000, 161, 31-48.

23 S. Rajesh, A. F. Ismail and D. R. Mohan, RSC Adv., 2012, 2, 6854-6870.

24 J. F. Li, Z. L. Xu, H. Yang, L. Y. Yu and M. Liu, Appl. Surf. Sci., 2009, 255, 4725-4732.

25 G. Wua, S. Gan, L. Cui and Y. Xu, Appl. Surf. Sci., 2008, 254, 7080-7086.

26 S. Singh, K. C. Khulbe and T. Matsuura, J. Membr. Sci., 1998, 142, 111-127.

27 A. Nagendran and D. Mohan, J. Appl. Polym. Sci., 2008, 110, 2047-2057.

28 M. Cheryan, Ultrafiltration Handbook, Technomic Publications Co., Lancaster, PA, USA, 1986.

29 J. Lee, W. Y. Ahn and C. H. Lee, Water Res., 2001, 35, 24352445.

30 M. A. Arguello, S. Alvarez, F. A. Riera and R. Alvarez, J. Agri. Food Chem., 2002, 50, 1951-1958.

31 X. Ma, C. Hu, R. Guo, X. Fang, H. Wu and Z. Jiang, Sep. Purif. Technol., 2008, 59, 34-42.
32 M. J. Zohuriaan and F. Shokrolahi, Polym. Test., 2004, 23(5), 575-579.

33 C. A. Smolders, A. J. Reuvers and R. M. Boom, J. Membr. Sci., 1992, 73, 259-275.

34 S. A. Mckelvey and W. J. Koros, J. Membr. Sci., 1996, 112, 2936.

35 W. R. Bowen and T. A. Doneva, J. Colloid Interface Sci., 2000, 229, 544-549.

36 M. Elimelech, X. Zhu, A. E. Childress and S. Hong, J. Membr. Sci., 1997, 127, 101-109.

37 A. Bessieres, M. Meireless, R. Coratger, J. Beauvillain and V. Sanchez, J. Membr. Sci., 1996, 109, 271-284.

38 A. Idris, N. M. Zaina and M. Y. Noordin, Desalination, 2007, 207, 324-339.

39 R. F. Brady Jr, Polymer Characterisation and Analysis, American Chemical Society, Oxford University Press, New York, 2003.

40 D. Murphy and M. N. Pinho, J. Membr. Sci., 1995, 106, 245257.

41 D. Y. Kwok, R. Lin, M. Mui and A. W. Neumann, Colloids Surf., A, 1996, 116, 63-77.

42 Z. Xu, X. Huang and L. Wan, Surface Engineering of Polymer Membranes, Springer, Zhejiang University Press, Berlin, Hangzhou, China, 2009.

43 W. R. Bowen, T. A. Doneva and J. G. Stoton, Colloids Surf., B, 2002, 27, 103-113. 